\title{
RELACIÓN CIRCULAR ENTRE ÉTICA, RESPONSABILIDAD SOCIAL Y REPUTACIÓN DE LAS COOPERATIVAS
}

\author{
POR \\ Isabel OLMEDO CIFUENTES ${ }^{1}$ \\ Inocencia María MARTÍNEZ LEÓN ${ }^{2}$ \\ Narciso ARCAS LARIO ${ }^{3}$ \\ Joaquín LONGINOS MARÍN RIVES ${ }^{4}$
}

\section{RESUMEN}

De forma progresiva, las empresas implantan programas y actividades sobre la base de la ética empresarial, la responsabilidad social y el buen gobierno, una estrategia justificada tanto por las actuales presiones procedentes de la sociedad como por los beneficios que obtienen en términos de reputación y de gestión de las relaciones con los stakeholders o grupos de interés. La propuesta de esta investigación es aplicar la dinámica en las relaciones de estas variables al caso de las cooperativas. Concretamente, se estudian las consecuencias de la ética empresarial y la responsabilidad social sobre la propensión a implantar códigos de conducta, y sus repercusiones sobre la consecución de un buen gobierno cooperativo y de una mayor reputación. Además, se razona que las cooperativas con mayor nivel de reputación tengan mayor tendencia a adoptar principios basados en la ética y la responsabilidad social. En definitiva, la principal aportación de este artículo se encuentra en la justificación de la relación circular existente entre estas variables para el caso de las cooperativas, en la medida en que son organizaciones con una singularidad propia en términos jurídicos, económicos y sociales.

\footnotetext{
${ }^{1}$ Universidad Politécnica de Cartagena. Dirección de correo electrónico: isabel.olmedo@upct.es

${ }^{2}$ Universidad Politécnica de Cartagena. Dirección de correo electrónico: ino.martinez@upct.es

${ }^{3}$ Universidad Politécnica de Cartagena. Dirección de correo electrónico: arcas.lario@upct.es

${ }^{4}$ Universidad de Murcia. Dirección de correo electrónico: longinos@um.es REVESCO No 107 - Primer Cuatrimestre 2012 - ISSN: 1885-8031 - www.ucm.es/info/revesco
}

DOI: $10.5209 /$ rev_REVE.2012.v107.38743

Fecha de recepción: 03/11/2010

Fecha de aceptación: 15/04/2011 
Palabras Clave: Ética empresarial, responsabilidad social, gobierno corporativo, códigos de conducta, reputación.

Claves ECONLIT: M100, M140, M190, P130.

\title{
CIRCULAR RELATIONSHIP AMONG ETHIC, SOCIAL RESPONSIBILITY AND REPUTATION OF COOPERATIVES
}

\begin{abstract}
Progressively, companies implement programs and activities based on business ethics, social responsibility and good governance. It is a justified strategy both in the current pressures from society, as the benefits that the firms obtain in terms of reputation and managing relationships with stakeholders. The proposal of this research is to apply the dynamics in the relationships of these variables to the case of cooperatives. In particular, it is studied the consequences of business ethics and social responsibility on the propensity to implement codes of conduct, and its impact on the achievement of good government in the cooperative and a greater reputation. Besides, it is argued that cooperatives with higher reputation are more likely to adopt principles based on ethics and social responsibility. In short, the main contribution of this paper is the justification for the circular relationship among these variables in the case of cooperatives, as they are organizations with an own singularity in terms of legal, economic and social.
\end{abstract}

Keywords: Business ethics, social responsibility, corporal citizenship, codes of conduct, reputation.

\section{INTRODUCCIÓN}

El desarrollo empresarial de los últimos años se ha visto empañado por una serie de escándalos económicos y financieros que han provocado que la sociedad exija a las organizaciones una mayor transparencia, ética y buen gobierno. Aunque estos aspectos puedan aparentar ser meros instrumentos de control y mantenimiento de buenas relaciones con los grupos de interés, se ha demostrado que son capaces de contribuir a mejorar la reputación corporativa, un activo intangible de indudable importancia para todo tipo de 
entidades empresariales (PriceWaterHouseCoopers, 2003; Carrasco, 2005; Valor, Merino y Palomo, 2005).

En la actualidad se están reclamando modelos de gestión empresarial que tomen en consideración los aspectos éticos, sociales y medioambientales de las actuaciones empresariales, así como los intereses de sus stakeholders. En este sentido, las entidades de economía social presentan una cierta ventaja respecto a las empresas mercantiles debido a que ya cuentan con una ideología propia de tipo participativo y democrático, mantenida y actualizada periódicamente en referencia a los valores y principios de la Alianza Cooperativa Internacional (Coque, 2005). Hay que considerar que las cooperativas tienen una especial singularidad económica, jurídica y social, que es recogida en la amplia normativa nacional, autonómica y sectorial sobre sociedades cooperativas analizada por Puentes, Velasco y Vilar (2009).

Gracias a la presente investigación, se avanza en la utilidad que puede implicar para las cooperativas la aplicación de prácticas relacionadas con la ética, la responsabilidad social, el gobierno corporativo o la reputación, en la medida en que pueden suponer un elemento de diferenciación y obtención de ventajas competitivas, que no sólo les reporten beneficios económicos sino también un equilibrio con el entorno en el que desarrollan su actividad.

Así, en situaciones de crisis como la actual, las cooperativas se presentan como una alternativa que fomenta la creación y mantenimiento del empleo y autoempleo ante un marco de desarrollo local. Por un lado, estas entidades desarrollan iniciativas empresariales únicas que responden a las necesidades de sus socios y a las de su entorno, favoreciendo el autoempleo. Por otro, sirven para ofrecer una relación laboral a sus trabajadores asalariados, generando empleo.

Esta situación actual justifica que el objetivo de esta investigación sea aportar una perspectiva teórica sobre cómo se intensifican las relaciones entre la ética empresarial, la responsabilidad social, el buen gobierno y la reputación en el ámbito de las cooperativas. Para ello, el presente trabajo se estructura de la siguiente manera. En primer lugar, se establece un marco general sobre las cooperativas, diferenciando sus características generales, sus principios de funcionamiento y los aspectos que se derivan de los mismos, pues son los elementos básicos sobre los que se realiza esta disertación. En segundo lugar, se estudia de 
forma pormenorizada lo que es la ética cooperativa, su relación con la responsabilidad social y cómo se reflejan ambas en los códigos de conducta de la entidad y en su buen gobierno. A continuación, se examina lo que se entiende por reputación, los beneficios organizativos que ésta proporciona y cómo se ha tratado de medir. Cuarto, a través de la revisión bibliográfica de la literatura realizada, se expone el objetivo de este trabajo, que es estudiar cómo se relacionan dichos conceptos en el ámbito de las cooperativas por dos razones iniciales; que todos ellos han sido considerados básicos en la obtención de ventajas competitivas empresariales en las últimas décadas, y que las peculiaridades de las cooperativas conducen a la idea de que estas entidades surgen con una ética y responsabilidad social innata. Dichos vínculos quedan reflejados en las hipótesis de estudio. Finalmente, se establecen las conclusiones del trabajo y las limitaciones del mismo.

\section{MARCO GENERAL DE LAS COOPERATIVAS}

Una cooperativa es una "sociedad constituida por personas que se asocian, en régimen de libre adhesión y baja voluntaria, para la realización de actividades empresariales, encaminadas a satisfacer sus necesidades y aspiraciones económicas y sociales, con estructura y funcionamiento democrático, conforme a los propios principios formulados por la Alianza Cooperativa Internacional" (Ley 27/1999: p. 4). Anteriormente, la Alianza Cooperativa Internacional $^{5}$ (1995) había definido la cooperativa como una asociación autónoma de personas que se han unido voluntariamente para satisfacer sus necesidades económicas, sociales y culturales comunes, mediante una empresa de propiedad conjunta y gestión democrática.

De estas definiciones se desprenden unas características generales de las cooperativas, tales como: a) son asociaciones de personas que tratan de responder por sí mismas a sus propias necesidades y/o a las de su entorno; b) sus miembros disfrutan de un régimen de libre adhesión y baja voluntaria; c) actúan en el mercado con la finalidad de producir, asegurar, financiar o distribuir bienes o servicios (Barea, 1990); d) son organizaciones democráticas controladas por sus miembros, quienes participan activamente en la definición de las políticas y en la toma de decisiones (Chaves, 1999); y e) integran la función económica y la función

\footnotetext{
${ }^{5}$ http://www.ica.coop/es/intro.html, (30/07/2010).
} 
social, pues crean riqueza con eficiencia económica y la distribuyen equitativamente entre los socios y la sociedad (Monzón, 1992).

Entre los principios de funcionamiento de las cooperativas destacan: a) su actividad empresarial se desarrolla como respuesta a ciertos fallos del mercado (precariedad del empleo, ausencia de servicios o necesidades sociales, culturales o económicas), a la necesidad de organizarse para tener un mayor poder de negociación frente a clientes y/o proveedores ${ }^{6} \mathrm{o}$ como una forma de desarrollo de las regiones y sectores más desfavorecidos fomentada por el Estado; b) el establecimiento democrático de unos objetivos comunes desde sus inicios (Chaves, 1999; Coque, 2008), destinados a satisfacer unas necesidades comunes de unos socios constituidos como un colectivo de emprendedores (Coque, 2008); c) los procesos de decisión son democráticos, pues las decisiones no se toman teniendo en cuenta el capital de cada socio, sino que los miembros tienen igual derecho de voto (cada socio tiene un voto), siendo la toma de decisión independiente al capital aportado por cada uno, a diferencia del sector privado capitalista donde impera la regla de un voto, una acción; d) la autonomía de gestión, diferenciándose con ello especialmente de las entidades pertenecientes a la esfera pública (Chaves, 1999); y e) la existencia de una primacía de las personas y el trabajo sobre el capital en el reparto de las rentas, de forma que la distribución de beneficios no está directamente vinculada con el capital aportado por cada miembro (Chaves, 1999), como revelan los criterios empleados por estas entidades ${ }^{7}$.

Como consecuencia de sus características y principios de funcionamiento, las cooperativas desarrollan: a) un principio de identidad único, delimitado por el hecho de que los socios son, a la vez, propietarios y usuarios de la cooperativa (Coque, 2008), lo que intensifica su interés en participar en la determinación de los objetivos de forma democrática y equitativa, eliminando intermediarios; b) unos vínculos sociales que refuerzan la dinámica asociativa y mercantil de la empresa dentro y fuera de la misma, otorgándole resistencia y flexibilidad en el mercado (Coque, 2008); c) un empleo más estable y creativo, en lugar de nuevas formas precarias del mercado de trabajo (Monzón, 1992); d) una actividad de innovación social y organizativa, en vez de ser agentes de parálisis (Monzón, 1992); e) unos principios que protegen el pluralismo y las preferencias de las minorías (Monzón, 1992); f)

\footnotetext{
${ }^{6}$ Acceso a nuevos canales de distribución, profesionales de alto nivel, economías de escala, nuevas tecnologías, o desarrollos de $\mathrm{I}+\mathrm{D}+\mathrm{i}$.

${ }^{7}$ Entre ellos, el principio cooperativo del retorno, la dotación de patrimonios colectivos, remuneración limitada al capital o la regla de no distribución de beneficios.
} 
una finalidad de servicio a sus miembros o a la colectividad antes que de lucro, enfatizando que la actividad económica desarrollada por estas entidades tiene vocación de servicio a personas y no de búsqueda del beneficio, aunque este último puede aparecer como un objetivo intermedio, puesto que la meta final de la organización es prestar el servicio; y g) una cultura empresarial propia, al compartirse una ideología sólidamente asentada en unos valores y principios cooperativos recogidos por la Alianza Cooperativa Internacional (Coque, 2008).

Precisamente, la ética específica con la que cuentan las cooperativas desde su constitución se fundamenta en una idiosincrasia comúnmente planteada en los principios cooperativos recogidos por la Alianza Cooperativa Internacional ${ }^{8}$ (1995), configuradores de la cultura empresarial de este tipo de entidades y que permiten poner en práctica una serie de valores y códigos de conducta. Estos principios cooperativos hacen referencia a: i) la adhesión voluntaria y abierta a todas las personas que quieran utilizar sus servicios y acepten la responsabilidad de ser un miembro de la misma, sin ningún tipo de discriminación; ii) la administración democrática por parte de los socios de la cooperativa, marcada por el principio de identidad de las cooperativas previamente mencionado; iii) la participación económica de los socios; iv) la autonomía e independencia de la organización, al ser controlada por sus propios miembros; v) la educación y formación de sus socios, representantes, directivos y empleados para contribuir al avance de la cooperativa, unida a la información transmitida sobre la naturaleza y beneficios de la cooperación; vi) la cooperación entre cooperativas, respecto al trabajo conjunto en estructuras locales, regionales, nacionales, e internacionales; y vii) la preocupación e interés por la comunidad, trabajando para lograr un desarrollo sostenible de las regiones en las que opera.

Estos principios cooperativos son los que definen la naturaleza y los cimientos de las cooperativas, de manera que cada organización los desarrollará de una forma concreta, dando lugar a una serie de códigos de conducta que regirán, entre otros aspectos, la forma de gobierno de la cooperativa, la actuación de la misma, su funcionamiento y su responsabilidad social frente a sus stakeholders (socios-propietarios, empleados, clientes, proveedores, y comunidad local donde desarrolla su actividad) (Belhouari, Buendía, Lapointe y Tremblay, 2005).

\footnotetext{
${ }^{8}$ http://www.ica.coop/es/intro.html, $(30 / 07 / 2010)$.
} 
En este sentido, cabe destacar que las cooperativas contribuyen a alcanzar una serie de beneficios como (Belhouari et al., 2005): a) favorecer un desarrollo sostenible en sus zonas de actuación; b) generar empleo; c) mejorar la integración y cohesión social; d) reforzar el sentimiento de pertenencia al territorio; e) reinvertir los beneficios en actividades primarias y secundarias en los lugares de origen, que evitan el éxodo de la población; y f) crear diversas infraestructuras socioeconómicas. Todo esto les permitirá diferenciarse del resto de organizaciones con el objeto de obtener ventajas competitivas.

\section{3. ÉTICA COOPERATIVA, RESPONSABILIDAD SOCIAL, CÓDIGOS DE CONDUCTA Y BUEN GOBIERNO}

\section{1. Ética y responsabilidad social en las cooperativas}

Para avanzar en el conocimiento de la ética empresarial es preciso analizar los planteamientos de la ética tradicional. Etkin (1993: p.16) afirma que la ética "es el fundamento cuyos valores esenciales deben organizar la vida social, y son tales como la libertad y la dignidad humana, así como también se basa en conceptos morales como el bien común, lo bueno, lo equitativo y lo justo". De este modo, la ética empresarial "es el descubrimiento y aplicación de los valores y normas compartidos por una sociedad pluralista valores que componen una ética cívica- al ámbito peculiar de la empresa, lo cual requiere entenderla según un modelo comunitario" (Cortina, 1994).

En consecuencia, la ética empresarial es una disciplina que trata de buscar la legitimidad y credibilidad de la labor empresarial (Valor et al., 2005), para crear confianza y cooperación con el entorno y con los grupos de interés (Palomo y Valor, 2001), puesto que no sólo se deben seguir las normas legales y del mercado, sino también las de la conciencia moral (González, 2001). Por ello, se puede definir como el modo en que la presión de la sociedad y, en concreto, de los stakeholders, fuerzan a la empresa a replantearse sus objetivos y medios empleados para alcanzarlos, reflexionando y asumiendo las consecuencias tanto positivas como negativas que surgen en el desarrollo de su actividad (González, 2001). Precisamente, García-Marzá (2007) establecía que la ética consiste en tener en cuenta las decisiones de todos los grupos implicados en la buena marcha de la empresa, para lograr la confianza necesaria y que se mantenga en el largo plazo. 
En el caso de las cooperativas, tal y como se ha visto en el segundo epígrafe de este trabajo, varios principios cooperativos determinan su actividad desde su creación, puesto que estas entidades desarrollan su labor al servicio del socio y de la comunidad, bajo una ética determinada por los principios cooperativos (Puentes et al., 2009).

Unido al concepto de ética empresarial, encontramos la responsabilidad social entendida como "el compromiso de una empresa de mantener un comportamiento corporativo autoexigente con todos sus stakeholders" (Villafañe, 2004: p. 62) o la integración voluntaria de las preocupaciones económicas, sociales y medioambientales en la dinámica operativa de las empresas sobre el marco de las relaciones con sus interlocutores (Comisión de las Comunidades Europeas, 2001). Otra definición es la ofrecida por De la Cuesta y Valor (2003: p. 7) que la entienden como "el conjunto de obligaciones y compromisos, legales y éticos, nacionales e internacionales, con los grupos de interés, que se derivan de los impactos que la actividad y operaciones de las organizaciones producen en el ámbito social, laboral, medio ambiental y de derechos humanos”. Así, una conducta socialmente responsable se asocia a la ética (Diller, 1999). Es decir, la ética empresarial recoge y analiza el marco general sobre la obligación moral de las organizaciones respecto a la sociedad; mientras que la responsabilidad social es una parte de la ética (Valor et al., 2005) que incluye el conjunto de acciones, decisiones y políticas que cada organización emplea para dar respuesta a las demandas y exigencias de sus grupos de interés (García-Marzá, 2007); definir hasta dónde llega esa responsabilidad (Cortina, 1994). Por tanto, la ética da sentido a la responsabilidad social (Cortina, 1994).

De acuerdo con lo expuesto, la responsabilidad social también es un aspecto inherente a la dinámica de funcionamiento de las cooperativas (Belhouari et al., 2005), pues las entidades de economía social integran los intereses de sus interlocutores y asumen de forma espontánea las responsabilidades sociales (Belhouari et al., 2005), lo cual se relaciona con un modelo de gobierno socialmente responsable o de stakeholders (Puentes et al., 2009).

Así, al crear una cooperativa, el gobierno de la misma planteará y formalizará una serie de directrices generales de comportamiento con base en la normativa específica ${ }^{9}$ y

\footnotetext{
${ }^{9}$ Cabe destacar que en España existe una normativa estatal sobre cooperativas, recogida en la Ley 27/1999, de 16 de junio, y una normativa a nivel autonómico referida ampliamente por los autores Puentes, Velasco y Vilar (2009: p. 131).
} 
general del sector (como las relacionadas con la protección del medio ambiente o calidad, entre otras) de aplicación a las cooperativas, unido a los principios cooperativos. Con posterioridad, y a medida que evolucione la entidad, se determinarán y fomentarán códigos de conducta más específicos sobre el comportamiento y responsabilidad de todos los cooperativistas en relación al resto de integrantes y al entorno en que desarrollan su actividad, promoviendo la toma de decisiones éticas en la organización (Valor et al., 2005). Precisamente, una ética y responsabilidad social integradas en la estrategia empresarial proporciona ventajas competitivas relacionadas con la reputación (Carrasco, 2005).

Antes de concluir este sub-epígrafe, es necesario puntualizar que existen otros factores o colectivos que pueden incidir en la ética y responsabilidad social de las cooperativas. Entre ellos destacan los cooperativistas, porque son quienes determinan en gran medida el grado en que la normativa de cumplimiento obligatorio, y la ética y responsabilidad social voluntarias, son aceptadas y ejercidas por la cooperativa. Sin embargo, este grupo de interés es abordado de forma generalista en este trabajo,

\subsection{Códigos de conducta y de buen gobierno. Aplicación a las cooperativas}

De forma general, un código de conducta es una declaración de principios y valores que una empresa formula por escrito para poner de manifiesto su propósito de atenerse a un comportamiento determinado, por lo que debe hacerse público fuera de ella (Diller, 1999). Los miembros de la dirección y gerencia son los encargados de establecer y difundir la ética y responsabilidad social que pondrá en práctica la empresa entre el resto de stakeholders. Así, la organización se compromete a respetar aspectos laborales (trabajadores, prácticas de proveedores contratados), sociales y medio ambientales, entre otros. Por tanto, tratan de reflejar la identidad y la cultura de la empresa, su ética y su responsabilidad que, en el caso de las cooperativas, ya viene implícita en sus características y principios de funcionamiento, como se ha indicado en epígrafes anteriores.

En consecuencia, los códigos que inciden sobre el gobierno de la cooperativa serán los que determinarán en buena medida su buen funcionamiento ético y responsable (Puentes et al., 2009), al ser este órgano el encargado de promover los valores de la cultura organizativa. 
Dentro de los códigos de conducta, es interesante analizar los códigos de buen gobierno. Por códigos de buen gobierno se puede entender un conjunto de recomendaciones acerca de la apropiada estructura, composición y funcionamiento de los órganos de gobierno y del adecuado comportamiento de los miembros de esos órganos respecto a los distintos grupos de interés, siendo el objetivo de estos códigos mejorar la transparencia informativa y dictar las directrices para instaurar un determinado modelo de gobierno corporativo (Aguilera y Cuervo, 2004; AECA, 2004). De este modo, los códigos de buen gobierno recogen tres aspectos clave (Valor et al., 2005): a) una guía de actuación honorable y profesional; b) las reglas básicas de organización y funcionamiento de una entidad; y c) las normas de conducta de sus miembros.

Partiendo de los códigos de conducta, hay que tener en cuenta una serie de condiciones en relación a la creación de los códigos de buen gobierno. En primer lugar, es necesaria una supervisión de la aplicación del código, pues a veces los buenos propósitos de los códigos de comportamiento no llegan a transcender de forma suficientemente efectiva, por no detallarse dónde y cómo deben verse reflejados en la gestión del día a día en la entidad. Por ello, un código de comportamiento debe ofrecer objetivos claros para orientar la relación de las entidades con todos sus stakeholders, ofreciendo principios visibles de actuación (Valor et al., 2005).

Por tanto, los códigos de buen gobierno fortalecen los sistemas de gestión, control y administración de las entidades para hacerlas más transparentes, eficientes y democráticas (Puentes et al., 2009). Se consideran un instrumento de gestión de las relaciones entre el principal y el agente (Crespí y Gisbert, 1999), teniendo en cuenta que pueden ser de dos tipos (Coque, 2003): a) entre propietarios y gestores, y b) entre socios directivos y el resto de socios. Además, los códigos de buen gobierno funcionan como mecanismos de autorregulación de las actuaciones de la organización y permiten, por un lado, formalizar la creación de valor para todos sus grupos de interés y, por otro, facilitar la obtención de confianza para el resto de grupos (Valor et al., 2005).

En la actualidad, no existe un código de buenas prácticas ajustado a la naturaleza y características de las sociedades cooperativas, lo que hace necesario elaborarlo o adaptar los ya existentes en otros ámbitos o sectores, tal y como concluían Puentes et al. (2009). Para ello, los principios cooperativos deben tomarse como una guía ética general (Valor et al., 
2005) y de responsabilidad social, a partir de la cual cada cooperativa debería crear su propio código de conducta y, por ende, de buen gobierno.

\subsection{El gobierno corporativo. Aplicación a las cooperativas}

La Organización para la Cooperación y el Desarrollo Económico (OCDE, 1999) define el término gobierno corporativo como el "sistema a través del cual los negocios son dirigidos y controlados; especificando la distribución de derechos y responsabilidades entre los diferentes participantes en la corporación, tal como el consejo, dirección, accionistas y otros grupos de poder; definiendo las reglas y procedimientos para la toma de decisiones en los temas corporativos; y proporcionando la estructura a través de la cual los objetivos de la compañía son fijados, así como los medios para lograr esos objetivos y controlar la rentabilidad".

La preocupación por el buen gobierno es generalizada en el ámbito empresarial, debido a (Spear, 2004): a) las excesivas competencias ejecutivas que culminan con remuneraciones desorbitadas a los directivos, prácticas de corrupción y una toma de decisiones pasiva ${ }^{10}$ por parte de este colectivo; b) el mayor interés por los sistemas a través de los cuales los propietarios ejercen su poder sobre los gerentes; c) los efectos de la globalización de las empresas y la relativa debilidad de los gobiernos nacionales en relación a ellas; y d) el creciente temor por los fracasos del mercado y por el medio ambiente, en relación a la propiedad común.

Desde el punto de vista de las cooperativas, el gobierno corporativo es aquel que se desarrolla a través de un sistema de normas, prácticas y procedimientos determinados de forma democrática ${ }^{11}$, en base a los cuales se regulan las relaciones que se producen en su interior y que tienen su reflejo en el exterior de la entidad. Por tanto, el gobierno de una cooperativa va a asumir tareas de gestión, organización, dirección y control de la actividad llevada a cabo, tratando de alinear los diferentes intereses de los stakeholders y, sobre todo, los de sus socios.

\footnotetext{
${ }^{10}$ Tal y como se ha puesto de manifiesto en las grandes corporaciones financieras de EEUU.

${ }^{11}$ Fundamentados en los derechos democráticos que se les concede a sus miembros para su gobierno (Spear, 2004).
} 
El objeto social de las cooperativas y su gestión democrática hace que sean más propensas a fijar criterios de buen gobierno, transparencia y responsabilidad social, superando a los criterios y principios existentes en el resto de sociedades (Palomo y Valor, 2004). Por otra parte, el buen gobierno trata de evitar problemas como la degeneración de las cooperativas, la tendencia a la estrechez de miras y las palancas políticas que fomentan el cambio en su estilo de gobierno (Spear, 2004). Por todo esto, estas entidades de economía social están ejerciendo, desde su creación, una filosofía empresarial que se ha anticipado a lo que la sociedad empieza a demandar (Palomo y Valor, 2004).

Además, en el buen gobierno inciden factores como (Chaves y Schediwy, 2004): a) las regulaciones gubernamentales impuestas por las autoridades; b) los conflictos de intereses entre los propietarios (accionistas/socios) y los controladores (directivos/gestores); o c) los códigos de conducta que garanticen: un buen gobierno con un amplio respaldo institucional y social (códigos de buen gobierno), el desarrollo y credibilidad de la actividad, la creación de una cultura organizativa y la adaptación al medio o entorno (Paz-Ares, 2008).

Por tanto, los códigos de buen gobierno son instrumentos que facilitan el gobierno corporativo. Sus características y aspectos clave recogidos en el sub-epígrafe anterior garantizan su influencia determinante en el gobierno cooperativo.

\section{LA REPUTACIÓN EMPRESARIAL EN LAS COOPERATIVAS}

La reputación corporativa o empresarial es un concepto de difícil definición debido a su formación a través de varias dimensiones (Weigelt y Camerer, 1988; Dollinger, Golden y Saxton, 1997; De Quevedo, 2003), relacionadas pero independientes (López e Iglesias, 2006; Martín y Navas, 2006); y su elevado grado de intangibilidad (Shenkar y Yuchtman-Yaar, 1997). Precisamente esta última característica es una de las causas de que la reputación sea considerada un activo intangible (Fombrun, 1996; Roberts y Dowling, 2002; Villafañe, 2004). Por ello, son numerosos los trabajos que lo clasifican como un recurso estratégico o una capacidad que reporta una serie de ventajas competitivas a las organizaciones (Fombrun y Shanley, 1990), lo que justifica la importancia que ha alcanzado en las últimas décadas, tanto en el ámbito académico como en el empresarial (Martínez y Olmedo, 2010). 
Partiendo de una perspectiva relacional ${ }^{12}$ (Chun, 2005), la reputación puede definirse como el conjunto de atributos económicos y no económicos vinculados a la empresa, que generan valor (Weigelt y Camerer, 1988) en base a una percepción representativa de la compañía (Fombrun, 1996). Esa apreciación estará influenciada por la actuación pasada de la empresa, las expectativas de futuro y la admiración que despierta entre sus rivales (Fombrun, 1996). Así, autores como Post y Griffin (1997: p. 165) definen el concepto de reputación como "una síntesis de opiniones, percepciones y actitudes de los grupos de interés de una organización, incluyendo empleados, clientes, proveedores, inversores y la comunidad". Esta definición está en la misma línea que la planteada por Ferguson, Deephouse y Ferguson (2000: p. 1196), quienes la entienden como "el conocimiento de las verdaderas características de una empresa y las emociones que hacia ella sienten los stakeholders o grupos de interés de la misma". Se trata, por tanto, de una valoración global que los grupos de interés realizan sobre una compañía en un momento determinado (Gotsi y Wilson, 2001).

En función de lo expuesto, la reputación empresarial depende de una serie de grupos de interés o stakeholders ${ }^{13}$, que en el caso las cooperativas serían los gestores, los socios, los empleados, los proveedores y los clientes, entre otros. Además, este activo influye significativamente en otras variables como la creación de valor (De Quevedo, 2003; Borraz y Fuentelsaz, 2005), y la generación de beneficios empresariales (Fombrun, Gardberg y Server, 2000; Wessels, 2003; Villafañe, 2004). Sus beneficios más destacados son (Martínez y Olmedo, 2010): la reducción de los costes de transacción, la contratación de trabajadores mejor preparados y en mejores términos, el acceso favorable al mercado de capitales y a las fuentes de financiación, un incremento de los precios, la reducción de la asimetría de la información, la creación de barreras de entrada a la competencia, la mayor lealtad por parte de los consumidores y usuarios, la posibilidad de diversificarse, y la llegada a clientes potenciales.

Las características de las cooperativas y su actividad empresarial no suponen, en principio, ninguna barrera para la obtención de los beneficios derivados de la reputación expuestos. Es por ello, que este activo permitirá mejorar los resultados empresariales (Teece, Pisana y Shuen, 1997), la solidez en el medio y largo plazo, y la creación de ventajas

\footnotetext{
12 Conjunto de apreciaciones o proceso de acumulación de información que los distintos grupos de interés realizan de una empresa.

${ }^{13}$ Grupos sin cuyo apoyo la organización dejaría de existir, pues influencian o son influenciados por la empresa, sus actividades y sus objetivos marcados.
} 
competitivas sostenibles en el tiempo (Barney, 1991; Grant, 1991; Hall, 1992; Amit y Schoemaker, 1993; Peteraf, 1993; Teece et al., 1997; Martín y Navas, 2006).

\section{RELACIÓN ENTRE ÉTICA, RESPONSABILIDAD SOCIAL, BUEN GOBIERNO Y REPUTACIÓN DE LAS COOPERATIVAS}

En general, la reputación empresarial es un activo intangible, fuente de ventajas competitivas, cuyos antecedentes son la ética, la responsabilidad social corporativa y el buen gobierno. Precisamente, para lograr una mayor transparencia en las actividades de la cooperativa y una mejora en el comportamiento y en la gestión de la misma, existen códigos de conducta que regulan las relaciones entre los agentes que conforman la entidad, destacando aquellos que se dirigen a conseguir un buen gobierno corporativo. Así, una percepción positiva de la ética, responsabilidad social y el gobierno de la cooperativa debe incidir directa y positivamente en su reputación frente a sus stakeholders.

Por otro lado, niveles de reputación aceptables en las organizaciones cooperativas deben influir en el desarrollo e implantación de la ética y la responsabilidad social corporativa, pues para salvaguardar los beneficios que genera la reputación, la entidad tratará de seguir manteniendo y mejorando sus comportamientos y prácticas para continuar siendo merecedora de la reputación lograda.

Por tanto, la relación teórica entre los conceptos destacados a lo largo de los epígrafes anteriores se puede plantear desde dos perspectivas: 1) las implicaciones de la ética cooperativa, responsabilidad social y el gobierno corporativo en la reputación, y 2) las implicaciones de la reputación de la cooperativa en la ética y la responsabilidad social de la entidad. Ambos vínculos permiten plantear la existencia de una relación circular entre la ética y la responsabilidad social respecto a la reputación, tal y como se representa gráficamente en la Figura 1. 
Figura 1. Relación circular de la ética, responsabilidad social, buen gobierno y reputación corporativa

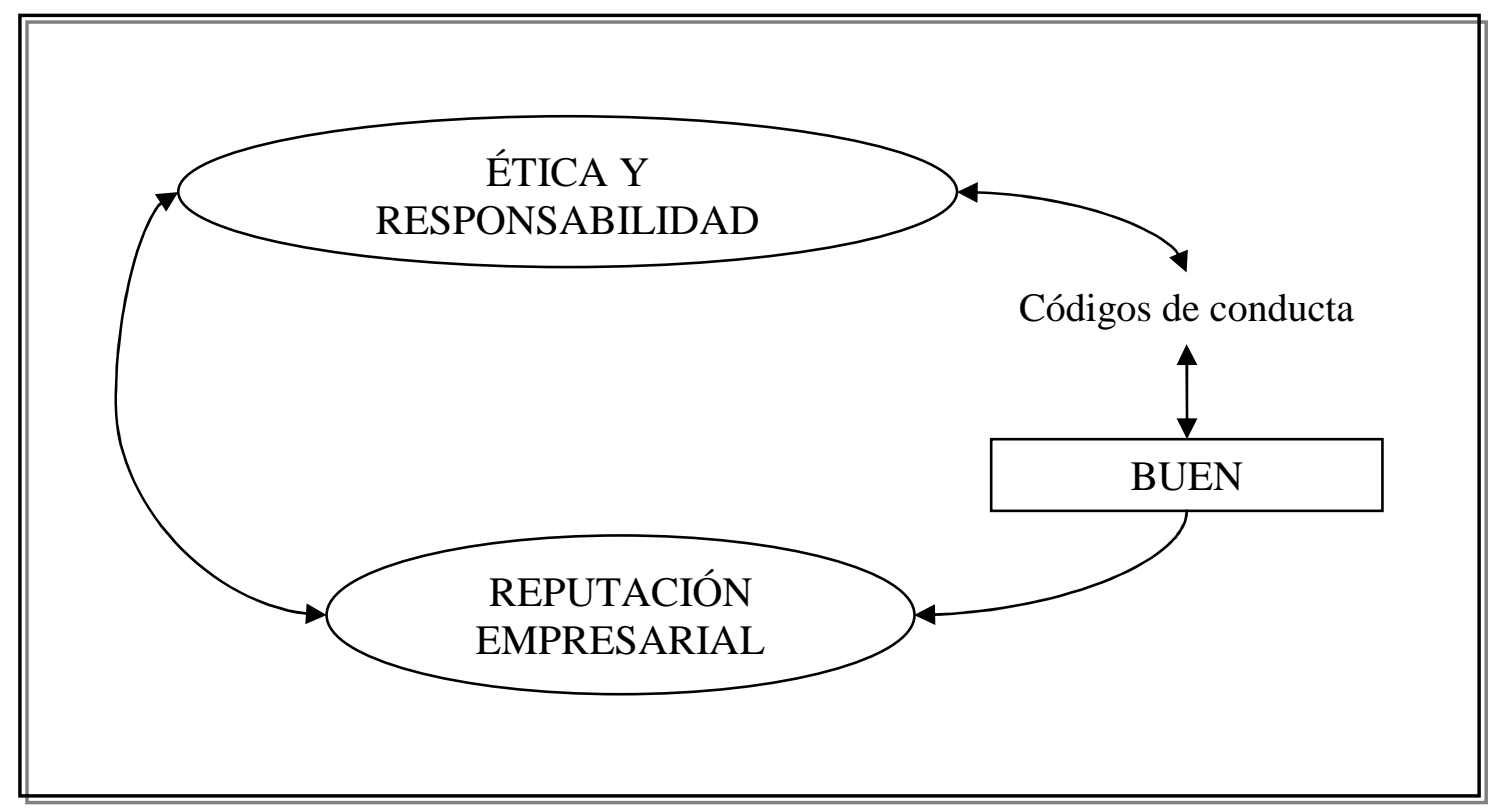

Fuente: Elaboración propia

\subsection{Implicaciones de la ética cooperativa, responsabilidad social y gobierno corporativo en la reputación}

Como ya se ha adelantado, las cooperativas se constituyen con una ética y responsabilidad "innatas”, de acuerdo a los principios cooperativos. Tanto la ética empresarial como la responsabilidad social se reflejan a través de los códigos de conducta en cualquier entidad. Dentro de esos códigos se encuentran los códigos de buen gobierno, que repercuten más allá de la propia organización, alcanzando intereses medioambientales y sociales de las comunidades en las que está enmarcada (como ocurre en el caso de las cooperativas). Los efectos de la utilización adecuada de dichos códigos de buen gobierno son dobles, pues por un lado, las entidades que tienen una firme voluntad de comportarse de forma ética al crear códigos, obtienen mejores resultados financieros (Valor et al., 2005); y por otro, los códigos de conducta también se utilizan para cumplir objetivos externos, como mejorar la reputación corporativa (Valor et al., 2005), evitar la regulación (Doig y Wilson, 1998; Kaye, 1996) o atraer inversiones (García de Madariaga y Valor, 2004).

En consecuencia, los códigos de buen gobierno tienen una gran repercusión sobre la reputación (Lizcano, 2002; Valor et al., 2005; Susaeta, Pin y Belizón, 2008), el desempeño de 
la entidad (Susaeta et al., 2008) y el éxito a largo plazo de la empresa (Lizcano, 2002); ayudando a las cooperativas a alcanzar ventajas competitivas en el entorno.

De este modo, parece que la ética y la responsabilidad social inciden positivamente en el buen gobierno de una entidad, pues cuando una cooperativa desarrolla códigos de conducta, tiene la intención de mostrar y regular la ética y responsabilidad social que va a guiar sus actuaciones, incluyendo las de su gobierno a través de los códigos creados a tal efecto. Por todo ello, parece razonable plantar la primera hipótesis:

H1. La ética y la responsabilidad social de las cooperativas están relacionadas de forma positiva con el buen gobierno corporativo, estando esta relación mediada por la influencia de los códigos de conducta.

Continuando el análisis de las relaciones entre variables, se puede considerar el gobierno corporativo un indicador clave de la buena gestión (Puentes et al., 2009), pues son numerosos los artículos e investigaciones que a lo largo de la literatura muestran su incidencia en la trayectoria empresarial y estudian la relación positiva que existe entre éste y los resultados obtenidos por las entidades (Gompers, Ishii y Metrick, 2003; Bauer, Günster y Otten, 2004; Bhagat y Bolton, 2008). En el caso de las cooperativas, los efectos positivos del gobierno corporativo se reflejan en la percepción interna y externa de los stakeholders, repercutiendo en consecuencia en su reputación. Además, no debemos de obviar que los factores básicos de los que depende el buen gobierno organizativo se fundamentan en los principios cooperativos, que se integrarán en la cultura organizativa de la entidad y en el carácter de los socios, debido a su capacidad de influir en el comportamiento ético y de responsabilidad social de la cooperativa a través de su voto, sus ideales y convicciones.

Puesto que la labor desarrollada por el gobierno cooperativo afecta tanto a la actuación pasada de la entidad como a su proyección futura, la percepción que se tenga de la reputación empresarial, de acuerdo a como se ha definido en el apartado cuarto, estará determinada por dicho gobierno. Esto nos lleva plantear la segunda hipótesis de este trabajo:

H2: El buen gobierno corporativo en las cooperativas tiene una influencia directa y positiva sobre la reputación. 
En este sentido, señalar que la apreciación de determinadas cooperativas en su ámbito de influencia (reputación) va a determinar su nivel o volumen de producción, como ocurre con las cooperativas de crédito o en las cooperativas de educación, donde los principios cooperativos que establezcan los socios van a ser fundamentales para el desarrollo de su actividad (Martínez y Olmedo, 2009).

\subsection{Implicaciones de la reputación de la cooperativa en la ética, responsabilidad social y buen gobierno cooperativo}

La reputación es un activo que genera una serie de beneficios (ya expuestos), cuya importancia relativa depende de cómo es percibida por los stakeholders vinculados a la cooperativa (clientes, empleados, proveedores y contexto social, entre otros), así como por sus competidores.

Por ello, las entidades deben diseñar sus actuaciones de forma que tengan en cuenta los intereses de todos o la mayoría de sus stakeholders, para mejorar su beneficio económico, social y medioambiental, siendo la ética y la responsabilidad social corporativa una de las herramientas de gestión de esas actuaciones (De la Cuesta, 2004; Vargas y Vaca, 2005; Server y Capó, 2009).

En esta línea, si la cooperativa es percibida como reputada por algunas de las características que los stakeholders tienen en cuenta, tales como la oferta de sus bienes y/o servicios, la imagen de marca, su nivel de innovación, o su posición financiera; se entiende que la ética y responsabilidad social de la entidad deben tener un nivel similar al admitido como normal por la sociedad, aunque nadie haya reparado explícitamente en él. Si no es así, la cooperativa puede encontrarse en situaciones tan difíciles como Kraft o Nestlé, cuya reputación se redujo drásticamente al conocerse que utilizaban un número considerable de esclavos infantiles en África occidental para la recolección de los frutos para el chocolate; o Nike, por las imputaciones sobre explotación, trabajo infantil, acoso sexual y otras irregularidades de sus empresas proveedoras; pese a que la valoración de sus productos es superior a la de otros de la competencia.

Ante esta situación, si la cooperativa ha alcanzado una buena reputación por razones ajenas a la ética y la responsabilidad social, no va a dejar que ambas sean razón para perderla; 
con lo cual va a trabajar en desarrollar los comportamientos éticos y socialmente responsables que le permitan mantenerse en el estatus reputacional conseguido, e incluso mejorarlo.

En otras situaciones bien diferentes, la ética y la responsabilidad social pueden emplearse como un reclamo en un primer momento (Cortina, 1994) y mejorar la imagen de la empresa. Si esas prácticas o políticas responsables no se afianzan, por ejemplo en una adecuada política de recursos humanos o la dirección y gestión de la cooperativa, esa apariencia de empresa ética y responsable se difuminará, pues la reputación es la imagen de una organización consolidada en el tiempo (Villafañe, 2004).

Así, decir que una empresa es ética y responsable genera buena reputación, pero si se queda sólo en una fachada, no consolida la reputación. Por tanto, una cooperativa con una reputación consolidada requiere el desarrollo y mantenimiento de un mayor compromiso público en cuanto a derechos humanos, laborales, medioambientales y de reducción de prácticas de corrupción, para poder mantener las expectativas creadas entre sus stakeholders.

Es por ello, que un nivel de reputación cooperativa aceptable por los stakeholders implica el desarrollo de una ética y responsabilidad social coherente con los principios cooperativos, en primer lugar, y con los códigos de buen gobierno, que favorecen la valoración positiva de la empresa en su entorno. Esta situación se debe a que los socios crean y mantienen una serie de valores en base a los principios cooperativos, que se integrarán en la cultura organizativa de la cooperativa así como en la percepción interna y externa que los grupos de interés posean de la misma, repercutiendo en consecuencia en su reputación.

Con esto, se justifica que la importancia de la ética y la responsabilidad social en la determinación de la reputación cooperativa será mucho más intensa que en cualquier otro tipo de entidad, como consecuencia de las características y principios de funcionamiento expuestos en el segundo epígrafe. En base a esta afirmación, se plantea que los stakeholders que valoren positivamente la reputación de una cooperativa, observarán posteriormente su ética y responsabilidad social en profundidad, estimando su nivel de implantación y, en su caso, exigiendo mayores niveles de desarrollo si la entidad no quiere perder su estatus reputacional. Esto permite pensar que las cooperativas con un buen nivel de reputación tendrán una ética y responsabilidad social desarrollada, implantada y conocida, fundamentada en los principios cooperativos, que irá evolucionando con la sociedad para mantener la 
reputación en el tiempo. Como consecuencia de los razonamientos anteriores se plantea la tercera hipótesis:

H3: La reputación de las cooperativas tiene un efecto directo y positivo sobre sus niveles de ética y responsabilidad social.

No obstante, esta reciprocidad no parece que teóricamente se pueda producir en el caso del buen gobierno, pues una cooperativa que se evalué como reputada, no se considera que a priori tenga que contar con un buen gobierno.

\section{CONCLUSIONES}

El estudio del vínculo entre la ética, la responsabilidad social, el buen gobierno y la reputación en el entorno de las cooperativas muestra que son conceptos muy cercanos, tal y como ocurre en las entidades de mercado. El objetivo que se pretende con este trabajo es mostrar la relación teórica circular que existe entre los mismos en el ámbito de las cooperativas.

Desde su constitución, las cooperativas cuentan con una normativa específica en relación al desarrollo de su actividad, de acuerdo a unos principios cooperativos especificados y planteados desde la Alianza Cooperativa Internacional. Todo esto se materializa en una ética y responsabilidad social, desarrollada a través de códigos de conducta que, a su vez, incidirán en el buen gobierno de la entidad y en la reputación empresarial. Por tanto, la ética, la responsabilidad social y el buen gobierno son componentes influyentes en la reputación de las cooperativas, siendo el nexo de unión los códigos de conducta y, en concreto, los códigos de buen gobierno.

Además, se plantea que las cooperativas que tengan una valoración positiva de su reputación querrán mantener esa posición mejorando sus actividades y políticas relacionadas con la ética y responsabilidad social, tal y como se ha expuesto. Como conclusión, se justifica teóricamente la existencia de una relación circular entre la ética y responsabilidad social respecto a la reputación. 
Es de interés puntualizar la inexistencia de reciprocidad en la relación entre buen gobierno y reputación empresarial, ya que ésta última depende de las percepciones de los stakeholders (calidad de los productos, innovación e imagen), que no siempre implican la existencia de un buen gobierno. Además, existen otros instrumentos que se utilizan para mantener y comunicar una transparencia informativa con el entorno en base a la ética y responsabilidad planteada por la cooperativa, que mejoran su reputación pero no implican un buen gobierno. Entre ellas destaca la publicación de memorias sociales, balances sociales, informes de sostenibilidad,..., etc. (Valor et al., 2005).

Este trabajo pretende que las cooperativas sean conscientes de la importancia que tienen sus características y principios de funcionamiento a la hora de competir con otras entidades. En concreto, su ética y responsabilidad social son una importante fuente de diferenciación, que por sus implicaciones en el buen gobierno y la reputación de la cooperativa, puede ser una potente ventaja frente a los competidores. Por ello, estas entidades deben considerar el hecho de fomentar el desarrollo de códigos de conducta (de acuerdo a los principios cooperativos), generalizar su uso y controlar el cumplimiento de los mismos para lograr intensificar las relaciones que se han expuesto en este trabajo.

Sin embargo, el presente trabajo adolece de una serie de limitaciones, como la necesidad de un estudio empírico que se plantee para un grupo de cooperativas concreto, como pudieran ser las dedicadas a la educación, por estar su volumen de negocio más ligado a la ética, la responsabilidad social, el gobierno y la reputación percibida por su entorno. Además, se podría plantear un estudio transversal para ver cómo evolucionan el conjunto de variables mencionadas para una serie de cooperativas, de distintos sectores, y ver qué acciones o medidas concretas afectan a todas ellas y a su óptima evolución. Otra limitación es que esta relación circular es muy específica para las cooperativas, por sus especiales condiciones sociales en su constitución y desarrollo. Esto hace que no esté claro que se pueda aplicar este modelo teórico a otras entidades de economía de mercado, puesto que su reputación puede no estar tan influenciada por la ética y responsabilidad social desarrollada, sino estar más afectada por otros aspectos como la calidad del producto o la imagen de la empresa. 


\section{BIBLIOGRAFÍA}

AGUILERA, Ruth y CUERVO-CAZURRA, Álvaro. Codes of good governance worldwide: what is the trigger?. Organizations Studies, Vol. 25, nº 3, 2004, p. 417-446.

ALIANZA COOPERATIVA INTERNACIONAL (ACI). Declaración de la Alianza Cooperativa Internacional sobre la Identidad Cooperativa: un informe. Anuario de Estudios Cooperativos. Universidad de Deusto, 1995, p. 71-97. Versión en español de: INTERNACIONAL COOPERATIVE ALLIANCE. The International Co-operative Alliance Statement on the Co-operative Identity, XXXI Congress International Cooperative Alliance, Manchester, United Kingdom, September, 20-22, 1995.

AMIT, Raphael y SCHOEMAKER, Paul J. H. Strategic assets and organizational rent. Strategic Management Journal, Vol. 14, nº 1, 1993, p. 33-46.

ASOCIACIÓN ESPAÑOLA DE CONTABILIDAD Y ADMINISTRACIÓN DE EMPRESAS (AECA). Marco conceptual de la responsabilidad social corporativa. Madrid: Comisión de Responsabilidad Social Corporativa - AECA, 2004, 84 p. ISBN: 978-84-89959-70-5.

BHAGAT, Sanjai y BOLTON, Brian. Corporate governance and firm performance. Journal of Corporate Finance, $\mathrm{n}^{\circ} 14,2008$, p. 257-273.

BAREA TEJEIRO, José. Concepto y agentes de la economía social. CIRIEC-España: Revista de debate de Economía Pública, Social y Cooperativa, no 8, octubre, 1990, p. 109-117.

BARNEY, Jay B. Firm resources and sustained competitive advantage. Journal of

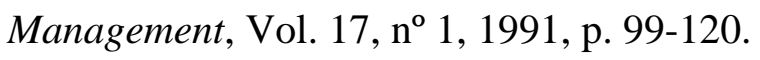

BAUER, Rob, GÜNSTER, Nadja y OTTEN, Roger. Empirical evidence on corporate governance in Europe: The effect on stock returns, firm value and performance. Journal of Asset Management, Vol. 5, $\mathrm{n}^{\circ}$ 2, 2004, p. 91-104.

BELHOUARI, Adil; BUENDÍA MARTÍNEZ, Inmaculada; LAPOINTE, Marie José y TREMBLAY, Benoit. La responsabilidad social de las empresas: ¿un nuevo valor para las cooperativas?. CIRIEC-España: Revista de Economía Pública, Social y Cooperativa, $\mathrm{n}^{\circ}$ 53, noviembre, 2005, p. 191-208.

BORRAZ MORA, Javier y FUENTELSAZ LAMATA, Lucio. La gestión del ciclo de vida de las capacidades: un análisis para el caso de la reputación. IV Iberoamerican Academy of Management, Lisboa, 8 - 11 diciembre, 2005.

CARRASCO, Inmaculada. La ética como eficiencia: la responsabilidad social en las cooperativas de crédito españolas. CIRIEC-España: Revista de Economía Pública, Social y Cooperativa, $\mathrm{n}^{\mathrm{o}}$ 53, noviembre, 2005, p. 351-367. 
CHAVES AVILA, Rafael. La economía social como enfoque metodológico, como objeto de estudio y como disciplina científica. CIRIEC-España: Revista de Economía Pública, Social y Cooperativa, $\mathrm{n}^{\circ} 33$, diciembre, 1999, p. 115-139.

CHAVES AVILA, Rafael y SCHEDIWY, Robert. Introducción al monográfico: Gobierno, democracia y directivos en las empresas cooperativas. CIRIEC-España: Revista de Economía Pública, Social y Cooperativa, no 48, abril, 2004, p. 5-10.

CHUN, Rosa. Corporate reputation: meaning and measurement. International Journal of Management Reviews, Vol. 7, $\mathrm{n}^{\circ}$ 2, 2005, p. 91-109.

COMISIÓN DE LAS COMUNIDADES EUROPEAS. Libro verde. Fomentar un marco europeo para la responsabilidad social de las empresas. Documento COM (2001) 366 final. Oficina de Publicaciones de las Comunidades Europeas. Luxemburgo.

CORTINA, Adela (cood.). Ética de la Empresa: claves para una nueva cultura empresarial. Madrid: Editorial Trotta, S.A., 1994. 150 p. ISBN: 9788481640131.

COQUE MARTÍNEZ, Jorge. De la eficiencia cooperativa: El gobierno participativo desde una perspectiva sistemática. Acciones e Investigaciones Sociales, Vol. 18, 2003, p. 67-87.

COQUE MARTÍNEZ, Jorge. Compartir soluciones: las cooperativas como factor de desarrollo en zonas desfavorecidas. Madrid: Comité Económico y Social (CES), 2005.

COQUE MARTÍNEZ, Jorge. Puntos fuertes y débiles de las cooperativas desde un concepto amplio de gobierno empresarial. REVESCO: Revista de Estudios Cooperativos, no 95 , 2008, p. 63-93.

CRESPÍ, Rafel y GISBERT, Carles. Block transfer. Implications for the governance of Spanish companies. II Foro de Finanzas, Segovia, 1999.

DE LA CUESTA GÓNZALEZ, Marta. El por qué de la responsabilidad social corporativa. Boletín ICE Económico, no 2813, 2004, p. 45-58.

DE LA CUESTA GÓNZALEZ, Marta y VALOR MARTÍNEZ, Carmen. Responsabilidad social de la empresa. Concepto, medición y desarrollo en España. Boletín Económico de ICE, $\mathrm{n}^{\circ} 2755,2003$, p. 7-19.

DE QUEVEDO PUENTE, Esther. Reputación y Creación de Valor. Una Relación Circular. $1^{\mathrm{a}}$ edición. Madrid: Paraninfo, 2003. ISBN: 8497322509.

DILLER, Janelle. ¿Una conciencia social en el mercado mundial? Dimensiones laborales de los códigos de conducta, el etiquetado social y las iniciativas de los inversores. Revista Internacional del Trabajo, Vol. 118, nº 2, 1999, p. 111-145.

DOIG, Alan y WILSON, John. The effectiveness of codes of conduct. Business Ethics: $a$ European Review, Vol. 7, no 3, 1998, p. 140-149. 
DOLLINGER, Mark J.; GOLDEN, Peggy A. y SAXTON, Todd. The effect of reputation on the decision to Joint Venture. Strategic Management Journal, Vol. 18, n 2, 1997, p. 127140.

ETKIN, Jorge R. La Doble Moral de las Organizaciones. Editorial McGraw-Hill Interamericana, 1993.328 p. ISBN: 8448101456.

FERGUSON, Tamela D.; DEEPHOUSE, David L. y FERGUSON, William L. Do strategic groups differ in reputation?. Strategic Management Journal, Vol. 21, n 12, 2000, p. 1193 1214.

FOMBRUN, Charles J. Reputation: realising value from the corporate image. Boston: Harvard Business School Press, 1996, 441 p. ISBN: 0875846335.

FOMBRUN, Charles J.; GARDBERG, Naomi A. y SERVER, Joy M. The reputation quotient: a multi-stakeholder measure of corporate reputation. Journal of Brand Management, Vol. 7, no 4, 2000, p. 241-255.

FOMBRUN, Charles J. y SHANLEY, Mark. What's in a name?: reputation building and corporate strategy. Academy of Management Journal, Vol. 33, nº 2, 1990, p. 233-258.

GARCÍA DE MADARIAGA MIRANDA, Jesús y VALOR MARTÍNEZ, Carmen. Analysis of the sustainable Business model among Spanish MNC's. Conference Proceedings of the Academy of Marketing, Virtual in Marketing, Cheltenham, 2004.

GARCÍA-MARZÁ, Domingo. Responsabilidad social de la empresa: una aproximación desde la ética empresarial. Veritas: Revista de Filosofía y Teología, n 17, 2007, p. 183-204.

GOMPERS, Paul A., ISHII, Joy L. y METRICK, Andrew. Corporate governance and equity prices, The Quarterly Journal of Economics, $\mathrm{n}^{\circ} 118,2003$, p. 107-155.

GONZÁLEZ ESTEBAN, Elsa. La responsabilidad moral de la empresa. Una revisión de la Teoría de Stakeholders desde la ética discursiva. Tesis Doctoral, Universidad Jaime I de Castellón, 2001.

GOTSI, Manto y WILSON, Alan M. Corporate reputation: seeking a definition. Corporate Communications, Vol. 6, no 1, 2001, p. 24-30.

GRANT, Robert M. The resource-based theory of competitive advantage: implications for strategy formulation. California Management Review, Vol. 33, no 3, 1991, p. 233-258.

HALL, Richard. The strategic analysis of intangible resources. Strategic Management Journal, Vol. 13, nº 2, 1992, p. 135-144.

KAYE, Bruce. Compliance and corporate culture: making the most out of codes of ethics. Australian Journal of Management, Vol. 21, $\mathrm{n}^{\circ}$ 1, 1996, p. 1-12. 
LEY 27/1999, de 16 de Julio, de Cooperativas. Boletín Oficial del Estado, no 170, p. 2702727062.

LIZCANO ÁLVAREZ, José Luís. Gobierno de las sociedades. Un enfoque desde la responsabilidad social. X Conferencia Anual de Ética, Economía y Dirección (EBENEspaña), Responsabilidad Social de las organizaciones. Madrid 13 y 14 de junio, 2002.

LÓPEZ LÓPEZ, Vicente A. e IGLESIAS ANTELO, Susana. Percepciones Directivas del Recurso Reputación. Estudio Empírico de sus Relaciones con el Rendimiento Empresarial. Cuadernos de Economía y Dirección de la Empresa, no 28, 2006, p. 139-160.

MARTÍN DE CASTRO, Gregorio y NAVAS LÓPEZ, José Emilio. La importancia de la reputación empresarial en la obtención de ventajas competitivas sostenibles. Investigaciones Europeas de Dirección y Economía de la Empresa, Vol. 12, nº 3, 2006, p. 29-39.

MARTÍNEZ LEÓN, Inocencia María y OLMEDO CIFUENTES, Isabel. Reputación y buen gobierno de las cooperativas. XII Jornadas Nacionales de Investigadores en Economía Social: La crisis como oportunidad para la Economía Social, Murcia, 24-26 junio, 2009.

MARTÍNEZ LEÓN, Inocencia María y OLMEDO CIFUENTES, Isabel. Revisión teórica de la reputación en el entorno empresarial. Cuadernos de Economía y Dirección de la Empresa, no 44, 2010, p. 59-77.

MONZÓN CAMPOS, José Luis. La economía social: Tercer sector de un nuevo escenario. En DEFOURNY, Jacques y MONZON, José Luís. Economía Social. Entre economía capitalista y economía pública. Valencia: CIRIEC-España editorial, 1992, p. 11-16. ISBN: 0213-8093.

ORGANIZACIÓN PARA LA COOPERACIÓN Y EL DESARROLLO ECONÓMICO (OCDE). Principios de la OCDE para el gobierno de las sociedades. Organización para la Cooperación y el Desarrollo Económico, París, 1999.

PAZ-ARES RODRÍGUEZ, José Cándido. El buen gobierno de las organizaciones no lucrativas (reflexiones preliminares). En PÉREZ DÍAZ, Víctor Miguel (Director). La filantropía: tendencias y perspectivas. Homenaje a Rodrigo Uría Meruéndano. Madrid: Fundación de Estudios Financieros, 2008, p. 147-158. ISBN: 978-84-612-528-2.

PALOMO ZURDO, Ricardo Javier y VALOR MARTÍNEZ, Carmen. Banca cooperativa: entorno financiero y proyección social. Madrid: UNACC - Unión Nacional de Cooperativas de Crédito, 2001, p. 328. ISBN: 84-932428-0-2

PALOMO ZURDO, Ricardo Javier y VALOR MARTÍNEZ, Carmen. El “activismo” de los socios como catalizador de la responsabilidad social de la empresa: aplicación a las 
organizaciones de participación. CIRIEC-España: Revista de Economía Pública, Social y Cooperativa, $\mathrm{n}^{\circ}$ 50, noviembre, 2004, p. 167-190.

PETERAF, Margaret A. The cornerstones of competitive advantage: a resource-based view. Strategic Management Journal, Vol. 14, nº 3, 1993, p. 179-191.

POST, James E. y GRIFFIN, Jennifer J. Corporate reputation and external affairs management. Corporate Reputation Review, Vol. 1, n 1/2, 1997, p. 165-171.

PRICEWATERHOUSECOOPERS. Responsabilidad social corporativa. Tendencias empresariales en España. Barcelona: PriceWaterHouseCoopers, 2003.

PUENTES POYATOS, Raquel; VELASCO GÁMEZ, María del Mar y VILAR HERNÁNDEZ, Juan. El buen gobierno corporativo en las sociedades cooperativas. REVESCO: Revista de Estudios Cooperativos, nº 98, 2009, p. 118-140.

ROBERTS, Peter W. y DOWLING, Grahame R. Corporate reputation and sustained superior financial performance. Strategic Management Journal, Vol. 23, $\mathrm{n}^{\mathrm{o}}$ 12, 2002, p. $1077-$ 1093.

SERVER IZQUIERDO, Ricardo J. y CAPÓ VICEDO, Jordi. La responsabilidad social empresarial en un contexto de crisis. Repercusión en las sociedades cooperativas. REVESCO: Revista de Estudios Cooperativos, ${ }^{\circ}$ 65, agosto 2009, p. 7-31.

SHENKAR, Oded y YUCHTMAN-YAAR, Ephraim. Reputation, Image, Prestige, and Goodwill: An Interdisciplinary Approach to Organizational Standing. Human Relations, Vol. 50, no 11, 1997, p. 1361-1381.

SPEAR, Roger. El gobierno democrático en las organizaciones cooperativas. CIRIECEspaña: Revista de Economía Pública, Social y Cooperativa, nº 48, abril, 2004, p. 11-30.

SUSAETA, Lourdes; PIN, José Ramón y BELIZÓN, María Jesús. Factores determinantes de la reputación del CEO: Un análisis sectorial entre las principales empresas españolas. IESE Business School - Universidad de Navarra. Noviembre, 2008. Documento de Investigación DI-772.

TEECE, David J.; PISANA, Gary y SHUEN, Amy. Dynamic capabilities and strategic management. Strategic Management Journal, Vol. 18, nº 7, 1997, p. 509-533.

VALOR MARTÍNEZ, Carmen; MERINO DE DIEGO, Amparo y PALOMO ZURDO, Ricardo Javier. Los códigos éticos en las cajas de ahorro y cooperativas de crédito: estado de la cuestión y análisis empírico. REVESCO: Revista de Estudios Cooperativos, $\mathrm{n}^{\circ} 85$, 2005, p. 61-85. 
VARGAS SÁNCHEZ, Alfonso y VACA ACOSTA, Rosa María. Responsabilidad social corporativa y cooperativismo: vínculos y potencialidades. CIRIEC-España: Revista de Economía Pública, Social y Cooperativa, no 53, noviembre, 2005, p. 241-260.

VILLAFAÑE, Justo. La Buena reputación. Clave del valor intangible de las empresas. Madrid: Pirámide, 2004. 194 p. ISBN: 9788436818390.

WEIGELT, Keith y CAMERER, Colin. Reputation and corporate strategy: a review of recent theory and applications. Strategy Management Journal, Vol. 9, nº 5, 1988, p. 443-454.

WESSELS, Catelijne. Reputation defined. CSR Magazine, Vol. 2/03, 2003, p. 28-29. http://www.csreurope.org/pages/en/csr_magazine.html (acceso 10 de octubre de 2010). 